\title{
DECEPTIVE CONVERGENCE IN MØLLER-PLESSET PERTURBATION ENERGIES
}

\author{
Peter M.W. GILL and Leo RADOM \\ Research School of Chemistry, Australian National University, Canberra, A.C.T. 2601, Australia
}

Received 16 September 1986

\begin{abstract}
Møller-Plesset perturbation calculations (MPn) up to fiftieth order, within both the restricted (RHF) and unrestricted Hartree-Fock (UHF) frameworks, have been used to examine the $\mathrm{He}_{2}^{2+}$ ground-state potential curve. The bond lengths of the equilibrium and transition structures have been optimized at all orders of perturbation theory. It is found that RMP $n$ describes the homolytic dissociation better than UMP $n$ for all $n>2$. This unexpected behaviour may be attributed to spin contamination in the UHF wavefunction. The UMP $n$ barriers deceptively appear convergent for small $n$ and the results may be indicative of dangers inherent generally in using the UMP approach with significantly spin-contaminated wavefunctions.
\end{abstract}

\section{Introduction}

One of the major contemporary procedures for incorporating electron correlation into molecularorbital calculations is the use of Møller-Plesset (MP) perturbation theory $[1,2]$. Because the MP expansion is truncated at some finite order, use of this procedure is predicated on a rapid convergence of the MP series.

Efficient methods have been devised for calculating the Møller-Plesset energy to second (MP2), third (MP3) or fourth (MP4) order [2,3] and it is of interest to enquire how close the MP4 results are to the limiting values. This has been done in a small number of cases by comparison with results of full configuration interaction (CI) calculations (see, for example, refs. $[4,5]$ ), but the latter are available for only a very limited number of molecules.

An alternative, less rigorous means of assessing convergence involves examining the changes that occur in progressing from Hartree-Fock (HF) (which is equivalent to MP1) to MP2 to MP3 to MP4. If these changes decrease in magnitude smoothly towards small values, this might be taken as an indication of a close approach to the limiting value.

One situation where the full CI result is readily accessible is for two-electron systems since, in these cases, full $\mathrm{CI}$ is achieved through straightforward
CISD (CI with all single and double excitations) calculations. The MP2, MP3 and MP4 results may also be obtained routinely and compared with the full CI result. We have found, in this manner, an example of deceptive convergence of the Møller-Plesset energies for one such two-electron system, namely the recently observed [6] dihelium dication $\left(\mathrm{He}_{2}^{2+}\right)$. Results relevant to this point are presented here.

In addition, recursion formulae have been derived which allow the evaluation of Møller-Plesset energies to any finite order for minimal-basis-set calculations on the $\mathrm{He}_{2}^{2+}$ system. This enables a critical examination of the convergence behaviour of restricted (RMP) and unrestricted Møller-Plesset (UMP) calculations.

\section{Method and results}

Using a modified version [7] of the GAUSSIAN 82 system of programs [8], standard ab initio calculations were carried out for $\mathrm{He}_{2}^{2+}$, both for the equilibrium structure and for the transition structure linking the molecule to two $\mathrm{He}^{+}$ions.

In an initial set of calculations, the equilibrium bond length in $\mathrm{He}_{2}^{2+}$ was optimized at each of twenty different levels of theory. These arise from combining successively each of four different basis sets with each of five different methods of incorporating elec- 
tron correlation. The basis sets used were:

(1) a minimal basis ${ }^{\ddagger}$ containing a single $s$ function on each helium atom $(3 \mathrm{~s}) \rightarrow[1 \mathrm{~s}]$;

(2) a double-zeta basis, 3-21G [9], with two s functions on each helium atom: $(3 \mathrm{~s}) \rightarrow[2 \mathrm{~s}]$;

(3) a double-zeta plus polarisation basis, $6-31 \mathrm{G}^{* *}$ [10] with two $s$ and a set of $p$ functions on each helium atom: $(4 \mathrm{~s} 1 \mathrm{p}) \rightarrow[2 \mathrm{~s} 1 \mathrm{p}]$;

(4) a "large" basis consisting of the 6-311G set which includes three s functions [11] supplemented by three sets of $p$ and a set of $d$ functions [12] on each of the helium atoms: (5s3p1d) $\rightarrow$ [3s3p1d].

These were used in conjunction with restricted Hartree-Fock theory (RHF), Møller-Plesset perturbation theory terminated at second, third and fourth order (RMP2, RMP3 and RMP4), and full configuration interaction $(\mathrm{CI})$.

In a second set of calculations, the bond length of the transition structure which leads to the dissociation of the dication into two monocations was obtained. The $\mathrm{He}_{2}^{2+}$ system is RHF $\rightarrow$ UHF unstable when the He-He distance exceeds about $0.85 \AA$ and since the transition structure has a bond length significantly greater than this, we can use either the restricted or unrestricted Hartree-Fock molecular

Formed by contraction of the 3-21G basis set, the coefficients being derived from a 3-21G calculation on $\mathrm{He}_{2}^{2+}$ at a bond length of $0.7 \AA$. orbitals (RHF and UHF, respectively) as the starting point for the Møller-Plesset perturbation calculations. We have explored both possibilities and, in so-doing, have calculated the bond length and energy of the transition structure at 36 diffcrent lcvels. These arise from all possible combinations of each of our four basis sets with each of RHF, RMP2, RMP3, RMP4, UHF, UMP2, UMP3, UMP4 and CI. The computed barriers at each of these levels of theory are summarized in tables 1 and 2.

The potential curve for $\mathrm{He}_{2}^{2+}$ has been accurately calculated via the James-Coolidge method by Yagisawa et al. [13]. Their results indicate that the curve possesses a local minimum at $r=0.70 \AA$ and a local maximum (the transition structure) at $r=1.15$ $\AA$ and that the encrgy difference between the two extrema is $145 \mathrm{~kJ} \mathrm{~mol}^{-1}$. We use these values as the standards by which to judge the predictions of each of the levels of theory which we have used.

Finally, we derived recursion formulae which enable us to calculate fifth-order and higher terms in the Møller-Plesset energy expansion for $\mathrm{He}_{2}^{2+}$ with an arbitrary minimal basis set. In this way, we have calculated (for our smallest basis set) RMPn and UMP $n$ potential curves for $\mathrm{He}_{2}^{2+}(0.55 \leqslant r \leqslant 1.45 \AA)$ for $n$ ranging from 2 to 50 , and have constructed an optimizer to locate the local minimum and maximum on each of these curves. Some of the results of these calculations are summarized in table 3 and fig. 1 .

Table 1

Calculated barrier heights using RHF orbitals $\left(\mathrm{kJ} \mathrm{mol}^{-1}\right)$

\begin{tabular}{llllrrr}
\hline Basis set & RHF & RMP2 & RMP3 & RMP4 & Full CI \\
\hline$(3 s)$ & $\rightarrow[1 s]$ & 924 & 313 & 145 & 96 & 102 \\
$(3 s)$ & $\rightarrow[2 s]$ & 610 & 191 & 82 & 46 & 33 \\
$(4 s 1 p) \rightarrow[2 s 1 p]$ & 794 & 337 & 199 & 147 & 130 \\
$(5 s 3 p 1 d) \rightarrow[3 s 3 p 1 d]$ & 805 & 345 & 210 & 157 & 139 \\
\hline
\end{tabular}

Table 2

Calculated barrier heights using UHF orbitals $\left(\mathrm{kJ} \mathrm{mol}^{-1}\right)$

\begin{tabular}{llllllr}
\hline Basis set & UHF & UMP2 & UMP3 & UMP4 & Full CI \\
\hline$(3 \mathrm{~s})$ & $\rightarrow[1 \mathrm{~s}]$ & 161 & 228 & 250 & 252 & 102 \\
$(3 \mathrm{~s})$ & $\rightarrow[2 \mathrm{~s}]$ & 108 & 160 & 175 & 173 & 33 \\
$(4 \mathrm{~s} 1 \mathrm{p}) \rightarrow[2 \mathrm{~s} 1 \mathrm{p}]$ & 164 & 237 & 256 & 257 & 130 \\
$(5 \mathrm{~s} 3 \mathrm{p} 1 \mathrm{~d}) \rightarrow[3 \mathrm{~s} 3 \mathrm{p} 1 \mathrm{~d}]$ & 166 & 251 & 266 & 266 & 139 \\
\hline
\end{tabular}


Table 3

Optimized bond lengths $(\AA)$ and barrier heights $\left(\mathrm{kJ} \mathrm{mol}^{-1}\right)$ at minimal-basis-set RMP $n$ and UMP $n$ levels

\begin{tabular}{|c|c|c|c|c|c|}
\hline \multirow[t]{2}{*}{$n$} & \multirow[t]{2}{*}{$r_{\mathrm{e}}$} & \multicolumn{2}{|l|}{ RMP } & \multicolumn{2}{|l|}{ UMP } \\
\hline & & $r_{\mathrm{TS}}$ & barrier & $r_{\mathrm{TS}}$ & barrier \\
\hline 1 & 0.6759 & 2.1434 & 924.1 & 0.9211 & 161.0 \\
\hline 2 & 0.6985 & 1.2497 & 312.5 & 0.9671 & 228.3 \\
\hline 3 & 0.7140 & 1.0809 & 145.1 & 0.9874 & 250.4 \\
\hline 4 & 0.7219 & 1.0284 & 96.3 & 1.0021 & 251.8 \\
\hline 5 & 0.7248 & 1.0211 & 85.5 & 1.0143 & 245.7 \\
\hline 6 & 0.7255 & 1.0362 & 88.4 & 1.0248 & 237.6 \\
\hline 7 & 0.7254 & 1.0643 & 95.9 & 1.0342 & 229.6 \\
\hline 8 & 0.7253 & 1.0957 & 102.9 & 1.0426 & 222.3 \\
\hline 9 & 0.7252 & 1.1015 & 105.7 & 1.0505 & 215.5 \\
\hline 10 & 0.7252 & 1.0884 & 105.0 & 1.0579 & 209.3 \\
\hline 11 & 0.7251 & 1.0775 & 103.5 & 1.0648 & 203.7 \\
\hline 12 & 0.7251 & 1.0716 & 102.4 & 1.0715 & 198.4 \\
\hline 13 & 0.7251 & 1.0699 & 101.9 & 1.0778 & 193.6 \\
\hline 14 & 0.7251 & 1.0707 & 101.8 & 1.0838 & 189.1 \\
\hline 15 & 0.7251 & 1.0727 & 102.0 & 1.0895 & 184.9 \\
\hline 16 & 0.7251 & 1.0745 & 102.2 & 1.0950 & 181.0 \\
\hline 17 & 0.7251 & 1.0752 & 102.3 & 1.1002 & 177.3 \\
\hline 18 & 0.7251 & 1.0750 & 102.4 & 1.1052 & 173.9 \\
\hline 19 & 0.7251 & 1.0744 & 102.3 & 1.1099 & 170.6 \\
\hline 20 & 0.7251 & 1.0740 & 102.3 & 1.1146 & 167.5 \\
\hline$\infty$ & 0.7251 & 1.0740 & 102.3 & 1.0740 & 102.3 \\
\hline
\end{tabular}

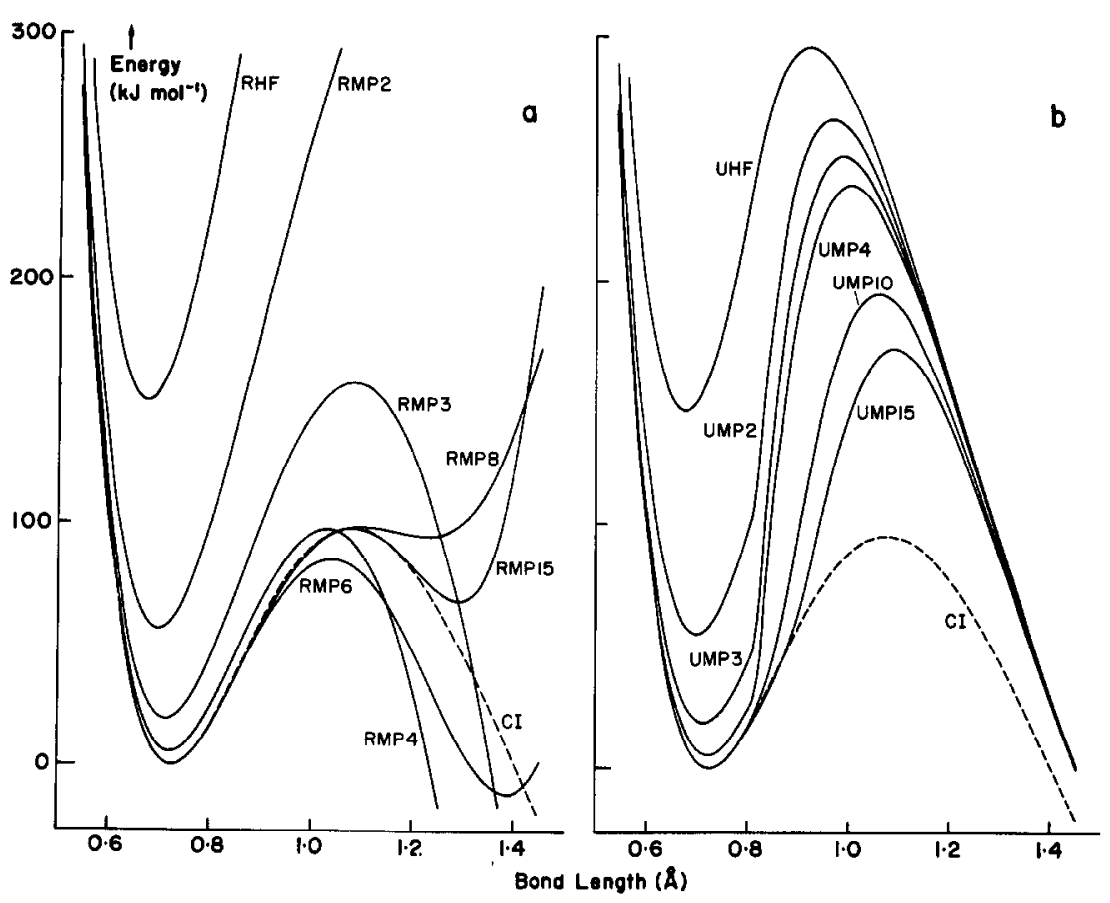

Fig. 1. Potential curves for $\mathrm{He}_{2}^{2+}$ at (a) minimal-basis-set $\operatorname{RMP} n(n=1,2,3,4,6$ and 8 ) and (b) minimal-basis-set UMP $n$ ( $n=1,2,3$, 4,10 and 15) levels. 


\section{Discussion}

Restricted Hartree-Fock theory gives a correct qualitative description of the heterolytic dissociation of $\mathrm{He}_{2}^{2+}$ into $\mathrm{He}+\mathrm{He}^{2+}$ but is incapable of correctly describing the lower-energy homolytic fission (to $\mathrm{He}^{+}+\mathrm{He}^{+}$) in which we are interested. In contrast, the unrestricted theory provides a qualitatively correct account of the energetics of both processes. However, when the RHF and UHF wavefunctions differ, only the former is an eigenfunction of the $S^{2}$ operator. The ground state $\left({ }^{1} \Sigma_{\mathrm{g}}^{+}\right)$of $\mathrm{He}_{2}^{2+}$ is a singlet, $\left\langle S^{2}\right\rangle=0$, for all internuclear distances through to the dissociation limit [13], but as the bond is stretched beyond $0.85 \AA$ the UHF wavefunction becomes progressively more and more contaminated by a (higher-energy) triplet state $\left({ }^{3} \Sigma_{u}^{+}\right)$with $\left\langle S^{2}\right\rangle$ $=2$. At infinite separation, where the RHF wavefunction is still a singlet, the UHF wavefunction has become an equal mixture of singlet and triplet states and consequently $\left\langle S^{2}\right\rangle=1$. It is important therefore to realize that while the UHF wavefunction "dissociates correctly" in an energetic sense, the wavefunction itself becomes a progressively poorer and poorer approximation as the bond length increases. That crucial distinction lies at the heart of this paper.

We see initially that full electron correlation, within a sufficiently large basis set, gives a satisfactory account of the barrier height. The values shown in tables 1 and 2 closely approach the result of Yagisawa et al. [13].

Restricted Hartree-Fock theory itself, even with our largest basis set, overestimates the barrier by more than a factor of five (table 1). However, when second-order perturbation theory based on these restricted orbitals is used, the barrier is reduced dramatically to less than half of the RHF value. Thirdand fourth-order treatments continue the improvement to the point where the RMP4 estimate of the barrier height differs from the full $\mathrm{CI}$ value by only $13 \%$.

Table 2 tells a very different story. The barrier is overestimated using unrestricted Hartree-Fock theory by about $20 \%$ with our best basis set and by larger proportions with the smaller sets. However, in stark contrast to the results of table 1 , second- and thirdorder perturbation treatments based on these UHF orbitals serve only to increase the estimate of the bar- rier height, and this peculiar effect occurs in a qualitatively similar fashion irrespective of which of our basis sets is used. The fourth-order correction is very small (0-2 $\left.\mathrm{kJ} \mathrm{mol}^{-1}\right)$ and it too is almost independent of the particular basis sct chosen. Morcover, so small are all the fourth-order corrections in table 2, and so smooth is the variation in barrier height from UHF to UMP4 (fig. 2), that it would be very tempting indeed to interpret these as evidence that the UMP series has converged and that, for example, the barrier using our largest basis set and a full correlation treatment is close to $266 \mathrm{~kJ} \mathrm{~mol}^{-1}$, when in fact it is only $139 \mathrm{~kJ} \mathrm{~mol}^{-1}$ !

The explanation for this deceptive convergence is clear from fig. $1 \mathrm{~b}$. In progressing from UMP3 to UMP4, the energies of the equilibrium and transition structures are lowered by almost exactly the same amount, so that the barrier height remains virtually unchanged. However, while almost all (96.5\%) of the minimal-basis-set correlation energy at the equilibrium structure has been recovered by the fourth-order treatment, a very much smaller fraction $(28.1 \%)$ has been recovered at the transition structure. The energy of the transition structure will therefore continue to drop as higher-order perturbation terms are included, leading ultimately to the substantial decrease in barrier height required to give the full $\mathrm{CI}$ result.

To what can we attribute these difficulties? In Møller-Plesset perturbation theory, the starting point

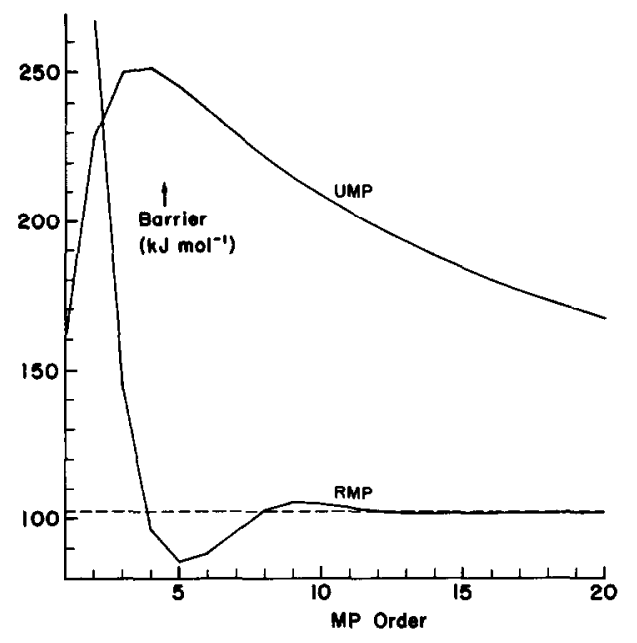

Fig. 2. Barriers to homolytic fission of $\mathrm{He}_{2}^{2+}$ using minimal-basisset MPn theory $(n=1-20)$. 
is a Hartree-Fock wavefunction, and the energy expansion will converge rapidly only if that wavefunction is sufficiently similar to the exact wavefunction. While the Hartree-Fock orbitals are a good starting point for $\mathrm{He}_{2}^{2+}$ at its equilibrium geometry, no single-determinant, restricted or unrestricted, offers a completely satisfactory zeroth-order wavefunction at the transition structure. The UMP perturbation series for the energy of the transition structure converges smoothly, but also slowly compared with that for the minimum that estimates of the barrier height drastically exaggerate it, even when many terms in the series are included. Conversely, as we shall see below, the RMP series for the transition structure, while converging much more quickly, does so in an oscillatory fashion. One consequence of this is that the sequence of RMP estimates of the barrier height also oscillates. Similar patterns of behaviour have been observed for the RMP and UMP expansions by Handy and co-workers [4] in the cases of stretched $\mathrm{H}_{2} \mathrm{O}$ and $\mathrm{NH}_{2}$ molecules.

These conclusions may be demonstrated quantitatively by an analytical examination of the convergence properties of both the restricted and unrestricted MP series beyond MP4. To do this requires non-standard methods since current stateof-the-art general-purpose programs such as GAUSSIAN 82 offer MP calculations only up to and including fourth-order. Although, in the general case, the calculation of higher-order energies is computationally very demanding and the programming of the specific formulae for the MP $n$ energies is extremely complex $[4,5]$, it turns out that, in the special case of a system with just two electrons and two basis functions, the task becomes comparatively simple. Fortunately, such a treatment is sufficient for our problem since the qualitative behaviour of MP theory with large basis sets is reproduced by our minimal set (tables 1 and 2). We have, in this way, been able to develop exact recursion formulae for the perturbation energies of $\mathrm{He}_{2}^{2+}$ with a minimal basis set. The computational effort required to evaluate the $n$ th-order perturbation energy using such formulae increases only linearly with $n$ compared with the exponential dependence on $n$ in the previously used, more general procedures $[4,5]$.

In a UHF framework, the interaction of two helium s orbitals results in two bonding spin orbitals $\psi_{1}^{\alpha}$ and $\psi_{1}^{\beta}$ (both with orbital energy $\epsilon_{1}$ ) and two antibonding spin orbitals $\psi_{2}^{\alpha}$ and $\psi_{2}^{\beta}$ (both with orbital energy $\left.\epsilon_{2}\right)$. The full correlation energy in this model is found as the lowest eigenvalue of the matrix

$\mathbf{H}=\left|\begin{array}{lll}0 & 0 & K_{2} \\ 0 & \alpha_{1}-x^{-1} & K_{1} \\ K_{2} & K_{1} & 2 \alpha_{2}-2 x^{-1}\end{array}\right|$,

which arises from expanding the $\mathrm{CI}$ wavefunction $|\Psi\rangle$ as a linear combination of the ground, (spinadapted) singly excited and doubly excited configurations

$$
|\Psi\rangle=\left|\Psi_{0}\right\rangle+c_{1}\left|\Psi_{1}\right\rangle+c_{2}\left|\Psi_{2}\right\rangle
$$

and then removing the Hartree-Fock energy. We use $K_{1}, K_{2}, \alpha_{1}$ and $\alpha_{2}$ to represent combinations of appropriate molecular integrals and $x=\left(\epsilon_{1}-\epsilon_{2}\right)^{-1}$.

The eigenvalue problem reduces to a cubic equation for the correlation energy $E$ :

$$
\begin{aligned}
& \left(1-\alpha_{1} x+x E\right)\left[x E^{2}+\left(2-2 \alpha_{2} x\right) E-K_{2}^{2} x\right] \\
& \quad=K_{1}^{2} x^{2} E,
\end{aligned}
$$

which, in the special case of RHF (where $K_{1}=0$ by symmetry), reduces to

$x E^{2}+\left(2-2 \alpha_{2} x\right) E-K_{2}^{2} x=0$.

The term in $x^{k}$ in the Maclaurin expansion of $E(x)$ is then the $(k+1)$ th-order perturbation correction to the Hartree-Fock energy, i.e.

$$
\begin{aligned}
E & =c_{1} x+c_{2} x^{2}+c_{3} x^{3}+\ldots \\
& =E_{2}+E_{3}+E_{4}+\ldots .
\end{aligned}
$$

The $E_{l}$ are most conveniently evaluated using recursion formulae. Defining

$$
\begin{aligned}
& T_{1}=\alpha_{1}+\alpha_{2}, \quad T_{2}=\frac{1}{2}\left(K_{1}^{2}+K_{2}^{2}\right)-\alpha_{1} \alpha_{2} \\
& T_{3}=\frac{1}{2} \alpha_{1}+\alpha_{2}, \\
& \gamma_{n}=\sum_{l=2}^{n-2} E_{l} E_{n-1}, \quad \delta_{n}=\sum_{i=2}^{n-4} E_{l} \gamma_{n-1}
\end{aligned}
$$

it eventually follows that the $n$ th-order Møller-Plesset correction to the energy is given by 


$$
\begin{aligned}
E_{n} & =x T_{1} E_{n-1}-\frac{3}{2} x \gamma_{n}+T_{2} x^{2} E_{n-2}+T_{3} x^{2} \gamma_{n-1} \\
& -\frac{1}{2} x^{2} \delta_{n}, \quad n>3,
\end{aligned}
$$

which, in the RHF case, becomes the much simpler

$E_{n}=x\left(\alpha_{2} E_{n-1}-\frac{1}{2} \gamma_{n}\right), \quad n>2$.

Using these formulae (once $K_{1}, K_{2}, \alpha_{1}, \alpha_{2}$ and $x$ are known for a chosen bond length), the terms in the RMP and UMP series become almost trivial to evaluate.

The results of table 3 show that the equilibrium bond length in $\mathrm{He}_{2}^{2+}$ is a very heavily damped oscillating function of $n$, the order of MP theory used. The value at MP4 differs from the full CI length by only $0.003 \AA$ and at MP5 this difference is reduced by another order of magnitude.

In contrast, the bond length in the transition structure either oscillates over a much greater range (in the RMP case), or progresses very slowly up to, beyond, and finally back to its limiting value (in the UMP case). For example, UHF gives a bond length which is $0.15 \AA$ too short, UMP4 still underestimates the CI value by more than $0.07 \AA$, the UMP 12 length is almost correct, and the UMP20 estimate is $0.04 \AA$ too long!

The barrier height (table 3 and fig. 2) plunges very rapidly over the first five orders of RMP theory and then oscillates with a rapidly decreasing amplitude about the full CI value. So-called "chemical accuracy" is attained above RMP7. However, with UMP theory, as we observed earlier, the barrier actually increases over the first four orders and only beyond UMP4 does it begin its extremely slow descent to the correct value. One unfortunate consequence of this unexpected behaviour is that the worst estimate by any order of UMP theory (including UHF itself) is that given by UMP4 and, compounding the problem, the fourth-order term $E_{4}$ is sufficiently small that, given only the barriers at levels up to and including UMP4, one could easily reach the false conclusion that convergence had been achieved.

The problems associated with the UMP treatment may undoubtedly be attributed to spin contamination in the UHF wavefunction. The $\left\langle S^{2}\right\rangle$ values in the vicinity of the transition structure are typically $0.5-0.8$ compared with the 0.0 required for a pure singlet. Spin-projected Møller-Plesset theory, as recently introduced by Schlegel [14], may offer a promising mechanism for overcoming the spin-contamination problem. In the case of our minimal-basis $\mathrm{He}_{2}^{2+}$ system, the full spin-projected formalism is particularly easy to apply since there is only triplet contamination, and such calculations are in progress.

\section{Conclusions}

Møller-Plesset perturbation theory provides a practically useful method of incorporating electron correlation only when the Hartree-Fock wavefunction upon which the theory is based is a sufficiently good zeroth-order approximation to the true wavefunction. In cases where a UHF wavefunction is significantly contaminated by states of higher multiplicities this proviso is not satisfied. In the example which we have studied, more accurate estimates of both geometries and barriers are obtained by using RMP $n$ uniformly along the entire potential curve than by using UMP $n$ for any $n$ greater than 2 . That this is found to be the case indicates that the "incorrect dissociation" behaviour of RHF does not impede the rapid convergence of the Møller-Plesset series as much as the presence of a triplet contaminant in the UHF wavefunction does. We believe that this is likely to be true in general and that caution should be exercised before applying UMP theory to heavily spin-contaminated wavefunctions.

\section{References}

[1] C. Møller and M.S. Plesset, Phys. Rev. 46 (1934) 618.

[2] J.A. Pople, J.S. Binkley and R. Seeger, Intern. J. Quantum Chem. Symp. 10 (1976) 1.

[3] J.A. Pople, R. Krishnan, H.B. Schlegel and J.S. Binkley, Intern. J. Quantum Chem. 14 (1978) 545;

R. Krishnan, M.J. Frisch and J.A. Pople, J. Chem. Phys. 72 (1980) 4244.

[4] N.C. Handy, P.J. Knowles and K. Somasundram, Theoret. Chim. Acta 68 (1985) 87, and references therein.

[5] W.D. Laidig, G. Fitzgerald and R.J. Bartlett, Chem. Phys. Letters 113 (1985) 15I, and references therein.

[6] M. Guilhaus, A.G. Brenton, J.H. Beynon, M. Rabrenovic and P. von R. Schleyer, J. Phys. B17 (1984) L605.

[7] J. Baker, R.H. Nobes, D. Poppinger and M.W. Wong, unpublished;

J. Baker, J. Comput. Chem., to be published. 
[8] J.S. Binkley, M.J. Frisch, D.J. DeFrees, K. Raghavachari, R.A. Whiteside, H.B. Schlegel, E.M. Fluder and J.A. Pople, GAUSSIAN 82, Carnegie-Mellon University, Pittsburgh, USA.

[9] J.S. Binkley, J.A. Pople and W.J. Hehre, J. Am. Chem. Soc. 102 (1980) 939.

[10] W.J. Hehre, R. Ditchfield and J.A. Pople, J. Chem. Phys. 56 (1972) 2257;

P.C. Hariharan and J.A. Pople, Theoret. Chim. Acta 28 (1973) 213.
[1 1 ] R. Krishnan, J.S. Binkley, R. Seeger and J.A. Pople, J. Chem. Phys. 72 (1980) 650.

[12] M.J. Frisch, J.A. Pople and J.S. Binkley, J. Chem. Phys. $80(1984) 3265$

[13] H. Yagisawa, H. Sato and T. Watanabe, Phys. Rev. A16 (1977) 1352.

[14] H.B. Schlegel, J. Chem. Phys. 84 (1986) 4530. 